\title{
ON NONAMENABLE GROUPS
}

\author{
SU-SHING CHEN \\ School of Mathematics \\ Georgia Institute of Technology \\ Atlanta, Georgia 30332 \\ U.S.A.
}

(Received Apri1 10, 1978 and in revised form October 3, 1978)

ABSTRACT. A sufficient condition is given for a countable discrete group $G$ to contain a free subgroup of two generators.

KEY WORDS AND PHRASES. Nonamenable group, free group.

AMS (MOS) SUBJECT CLASSIFICATION (1970) CODES. 22005.

Given a topological group $G$, we denote by $L$ the Banach algebra of all real valued bounded left uniformly continuous functions on G with the supremem norm. A mean $\mathrm{m}$ on $\mathrm{L}$ is a continuous, positive, linear functional such that $\mathrm{m}(1)=1$. A mean is called invariant if $m\left(f^{g}\right)=m(f)$ for every $f \in L$ and $g \in G$, where $f^{g}$ is the translate of $\mathrm{f}$ by $\mathrm{g}$.

$G$ is called amenable if there exists an invariant mean on $L$. $G$ has the fixed point property if whenever $G$ acts on a compact convex set $Q$ affinely in a locally convex topological vector space $E$, then $G$ has a fixed point in $Q[2]$. 
It is well known that $G$ is amenable if and only if $G$ has the fixed point property for any topological group G.

In [4], von Neumann proved that if $G$ has a free subgroup of two generators then $G$ is not amenable and conjectured that the converse is true. In this paper, we shall give a sufficient condition for a discrete group $G$ to contain a free subgroup of two generators. This result may be interesting to the investigation of von Neumann's conjecture.

Let $\phi$ be an affine transformation of a compact convex set $Q$ in a locally convex topological vector space $\mathrm{E}$. Then $\phi$ has a fixed point in $Q$ by the famous Tychonoff fixed point theorem. Furthermore, one can prove easily that the fixed point set $F_{\phi}$ of an affine transformation $\phi$ of $Q$ is a compact convex subset of $Q$.

Let us consider a discrete group $G$ acting affinely on $Q$. The fixed point set $F_{\phi}$ of each element $\phi$ of $G$ coincides with the fixed point set $F_{\phi}-1$ of the inverse $\phi^{-1}$. An element $\phi$ of $G$ is said to be attractive if for each weak neighborhood $U_{\phi}$ of the fixed point set $F_{\phi}$ of $\phi$, the orbit $\left\{\phi^{n}(S) \mid n \in \mathbb{Z}\right\}$ of any compact convex subset $S$ in $Q-U_{\phi}$ converges to the fixed point set $F_{\phi}$ of $\phi$, that is, there is a positive integer $N$ such that for all $|n|>N, \phi^{n}(S) \subset U_{\phi}$. An element $\phi$ of $G$ is said to be weakly attractive if, for each weak neighborhood $U_{\phi}$ of the fixed point set $F_{\phi}$ of $\phi$, there is a positive integer $N^{\prime}$ such that for all $\mathrm{n} \in \mathbb{Z}^{*(1)}, \phi^{\mathrm{nN}^{\prime}}(\mathrm{S}) \subset \mathrm{U}_{\phi}$. It is obvious that an attractive element $\phi$ of $\mathrm{G}$ is weakly attractive. [Note: (1) $\mathbb{Z}^{*}=\mathbb{Z}-\{0\}$ ]

THEOREM. If a discrete group $G$ acts on a compact convex set of $Q$ of a locally convex topological vector space $E$ affinely such that $G$ contains at least two weakly attractive elements without common fixed points, then G contains a free subgroup of two generators.

PROOF. Let $\phi$ and $\psi$ be two weakly attractive elements of $G$. Then the fixed point sets $F_{\phi}$ and $F_{\psi}$ are disjoint. By the seperation theorem [6], there exist 
a IInear functional $L$ on $E$ and real numbers $c_{1}$ and $c_{2}$ such that $L x<c_{1}<c_{2}<$ Ly for every $x$ in $F_{\phi}$ and every $y$ in $F_{\psi}$. Without loss of generality, we may assume that $c_{1}<0<c_{2}$.

Thus $K_{1}=\{x \in Q \mid L x<0\}$ is a weak convex neighborhood of $F_{\phi}$ and $\mathrm{K}_{2}=\{\mathrm{x} \in \mathrm{Q} \mid \mathrm{Lx}>0\} \quad$ is a weak convex neighborhood of $\mathrm{F}_{\psi}$. The complements $\mathrm{K}_{1}^{\mathrm{c}}$ and $K_{2}^{c}$ of $K_{1}$ and $K_{2}$ respectively are compact and convex sets in $Q-K_{1}$ and $Q-K_{2}$. By the definition of weak attractiveness, there exist positive integers $N^{\prime}$ and $N^{\prime \prime}$ such that $\phi^{\mathrm{nN}^{\prime}}\left(\mathrm{K}_{1}^{\mathrm{c}}\right) \subset \mathrm{K}_{1}$ and $\psi^{\mathrm{nN}}\left(\mathrm{K}_{2}^{\mathrm{c}}\right) \subset \mathrm{K}_{2}$ for all $\mathrm{n} \in \mathbb{Z}^{*}$. Let $s=\phi^{\mathrm{N}^{\prime}}$ and $t=\psi^{N}$. Then the group $F$ generated by $s$ and $t$ is a free group. In fact, for any relation $s^{p_{t}} \ldots=$ id, we have $s^{p_{t}} \ldots(z)=z$ for each $z$ in the hyperplane section $K_{1}^{c} \cap K_{2}^{c}=\{z \in Q \mid L z=0\}$ of $Q$. But clearly Ls ${ }^{p_{t}}{ }^{q} \ldots(z) \neq 0$, while $\mathrm{Lz}=0$. We have a contradiction.

COROLLARY. If a nonamenable discrete group $G$ acts on a compact convex set $Q$ of a locally convex topological vector space $E$ affinely such that $G$ contains all weakly attractive elements then $G$ contains a free subgroup of two generators.

PROOF. This follows from the theorem and the non-fixed point property of nonamenable groups.

ACKNOWLEDGEMENT. The author is indebted to the referee for his comments. 


\section{REFERENCES}

1. Furstenberg, H., A Poisson Formula for Semisimple Lie Groups, Ann. of Math. 77 (1963) 335-383.

2. Glasner, S., Proximal Flows, Lecture Notes in Math., Springer-Verlag, Vo1. $517,1976$.

3. Greenleaf, F. P., Invariant Means on Topological Groups, Van Nostrand Math. Studies, 1969.

4. Klee, V., Some Topological Properties of Convex Sets, Trans. Amer. Math. Soc., $\underline{78}$ (1955) $30-45$.

5. Von Neumann, J., Zur allgemeinen theorie des masses, Fund. Math. $\underline{13}$ (1929) 73-116.

6. Rudin, W., Functional Analysis, McGraw-Hill 1973.

7. Tits, J., Free Subgroups in Linear Groups, Jour. of Algebra 20 (1972) 250-270. 


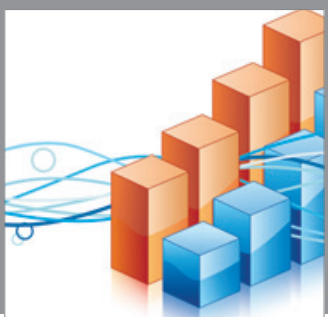

Advances in

Operations Research

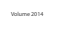

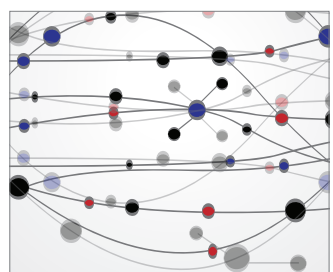

\section{The Scientific} World Journal
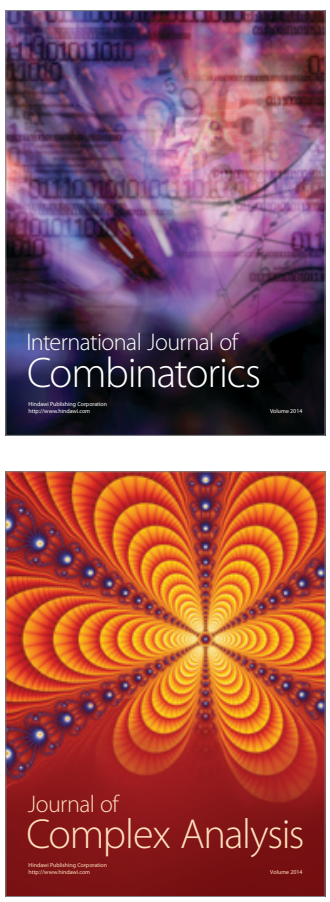

International Journal of

Mathematics and

Mathematical

Sciences
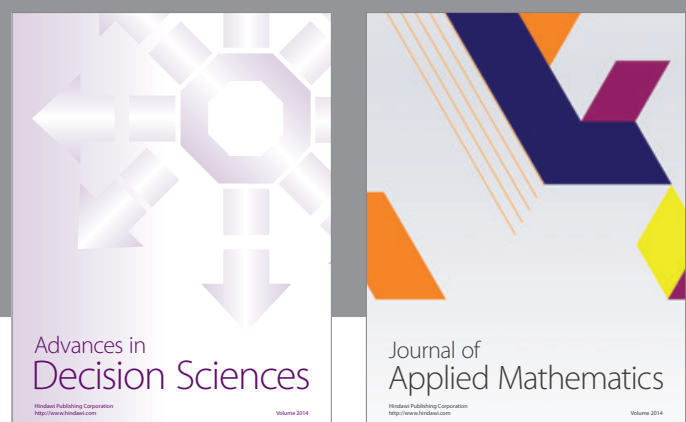

Journal of

Applied Mathematics
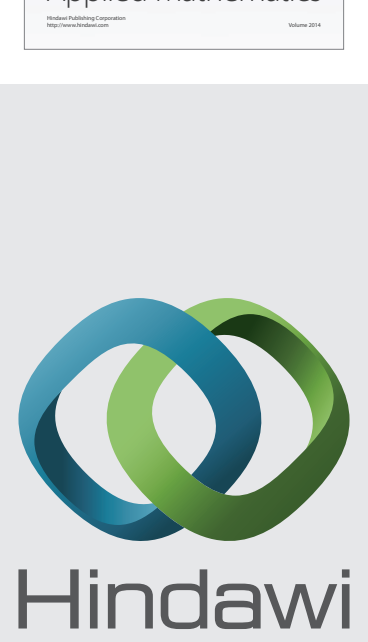

Submit your manuscripts at http://www.hindawi.com
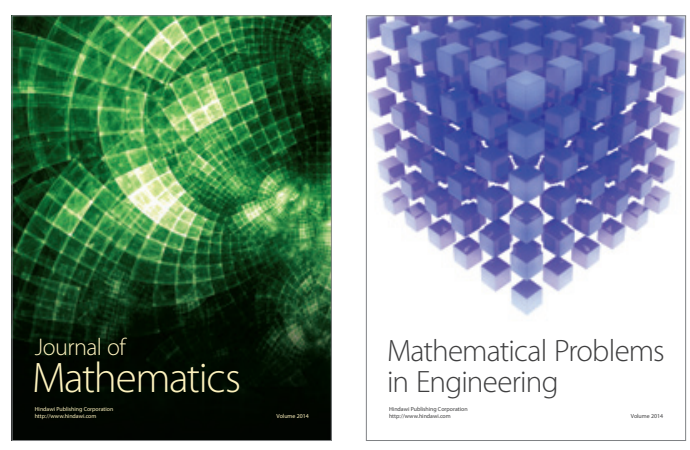

Mathematical Problems in Engineering
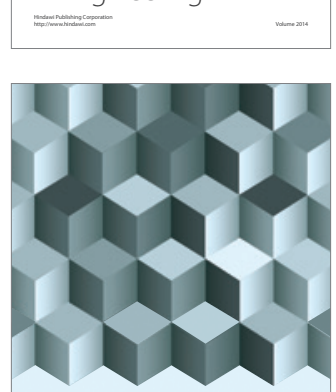

Journal of

Function Spaces
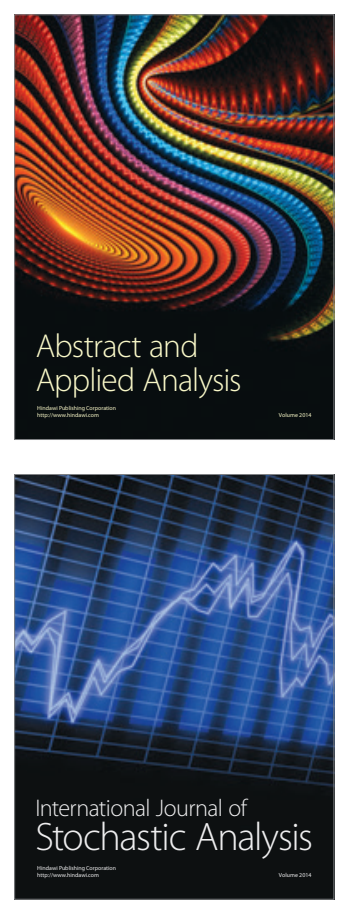

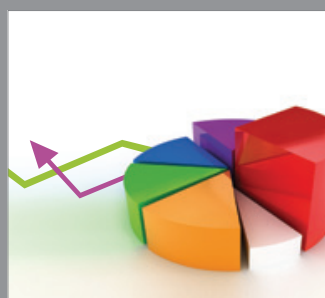

ournal of

Probability and Statistics

Promensencen
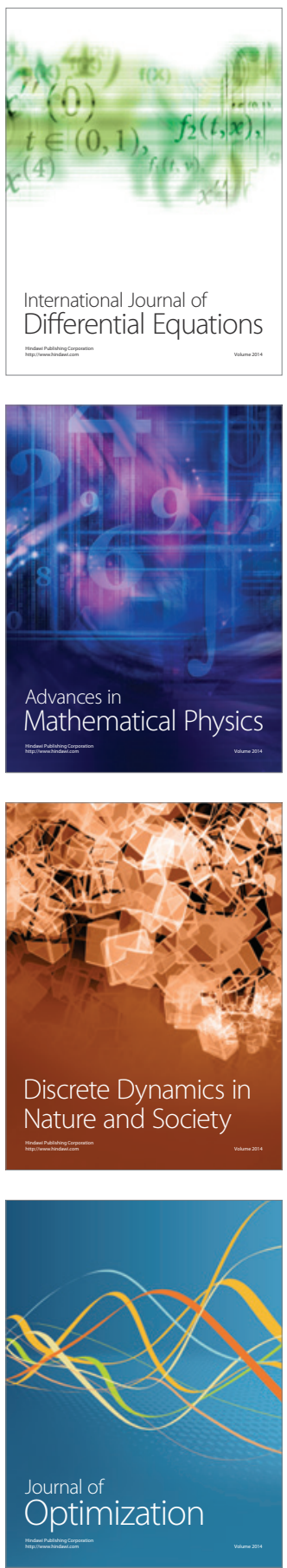